\title{
Re-affirming the connection between the Galactic stellar warp and the Canis Major over-density
}

\author{
M. López-Corredoira ${ }^{1}$, Y. Momany ${ }^{2}$, S. Zaggia ${ }^{2}$, and A. Cabrera-Lavers ${ }^{1,3}$ \\ ${ }^{1}$ Instituto de Astrofísica de Canarias, C/.Vía Láctea, s/n, 38200 La Laguna (S/C de Tenerife), Spain \\ e-mail: martinlc@iac.es \\ 2 INAF - Oss. Astronomico di Padova, Vicolo dell'Osservatorio 5, 35122 Padova, Italy \\ GTC Project Office, C/.Vía Láctea, s/n, 38200 La Laguna (S/C de Tenerife), Spain
}

Received 8 May 2007 / Accepted 27 July 2007

ABSTRACT

\begin{abstract}
Aims. We aim to understand the real nature of the stellar overdensity at southern galactic latitudes in the region of CMa. Methods. We perform a critical re-analysis and discussion of recent results presented in the literature which interpret the CMa overdensity as the signature of an accreting dwarf galaxy or a new substructure within the Galaxy. Several issues are addressed. Results. We show that arguments against the "warp" interpretation are based on an erroneous perception of the Milky Way. There is nothing anomalous with colour-magnitude diagrams on opposite sides of the average warp mid-plane being different. We witnessed the rise and fall of the blue plume population, first attributed to young stars in a disrupting dwarf galaxy and now discarded as a normal disc population. Similarly, there is nothing anomalous in the outer thin+thick disc metallicities being low $(-1<[\mathrm{Fe} / \mathrm{H}]<-0.5)$, and spiral arms (as part of the thin disc) should, and do, warp. Most importantly, we show unambiguously that, contrary to previous claims, the warp produces a stellar overdensity that is distance-compatible with that observed in CMa.

Conclusions. The CMa over-density remains fully accounted for in a first order approach by Galactic models without new substructures. Given the intrinsic uncertainties (concerning the properties of the warp, flare and disc cutoff, the role of extinction and degeneracy), minor deviations with respect to these models are not enough to support the hypothesis of an accreted dwarf galaxy or new substructure within the Milky Way disc.
\end{abstract}

Key words. Galaxy: structure - galaxies: dwarf

\section{Introduction}

Since its discovery (Martin et al. 2004), the Canis Major (CMa) over-density of stars has been the subject of a lively debate over whether it is a dwarf galaxy or simply the warped/flared Galactic disc (see Momany et al. 2006 (hereafter M06); López-Corredoira 2006 (hereafter L06), and references therein). However, there are many alternative "solution" papers (for and against the dwarf galaxy origin). In the absence of a clear-cut evidence in favour of an extra-Galactic origin (e.g. chemical enrichment), attention was focused on the star counts and stellar populations of the CMa over-density. In this regard, the new wide-field surveys by Conn et al. (2007, hereafter C07), Butler et al. (2007, hereafter B07) and de Jong et al. (2007, hereafter d07) presented deep colour-magnitude diagrams (CMDs) that, in principle, challenge the warp hypothesis. In this paper, we explain why this is not the case.

We do not explain all the second order details of the $\mathrm{CMa}$ overdensity solely in terms of the warping/flaring of the Milky Way stellar disc. Momany et al. (2004), M06 and L06 demonstrated that, on the basis of its star counts, the CMa over-density cannot unambiguously be disentangled from the warp feature, and that a Galactic origin, given the uncertainties in all Galactic models, remains the first-order explanation. Our purpose is to reply to the claims of $\mathrm{C} 07, \mathrm{~B} 07$ and d07, critically re-analysing some of their results and conclusions, in the light of the "warp" solution and demonstrating that it is still the most plausible one to explain the CMa stellar overdensity.

\section{Critical re-analysis of the $\mathrm{CMa}$ overdensity}

In the following subsections we show that the observational data of C07, B07 and d07 can be better explained by normal Milky Way warped disc modelling than by a dwarf galaxy or new substructure in the Galaxy. In particular, erroneously interpreted or wrongly used aspects will be pointed out.

\subsection{CMDs on opposite sides of the warp}

The main result of the $\mathrm{C} 07$ and B07 surveys (and major objection to the warp interpretation) is that CMDs of the centre of CMa and control northern hemisphere fields show different morphology and star counts. Their large survey coverage allowed a CMD comparison not only for opposite hemisphere fields at different latitudes but also for fields equally distant from the nominal warped mid-plane. The details of this CMD comparison are found in B07 (Sect. 7.2, Fig. 5) $\left(b \approx+8^{\circ}\right.$ vs. $\left.b \approx-15^{\circ}\right)$ and $\mathrm{C} 07$ (Sect. 6.2.1, Fig. 28) $\left(b \approx+4^{\circ}\right.$ vs. $\left.b \approx-9^{\circ}\right)$. Both groups attribute the differences to the presence of an extra stellar population (in addition to that of the warp). B07 and C07's conclusions were based on the finding by M06 (Sect. 4.2) that the mid-plane for Galactic longitudes of $l \sim 240^{\circ}$ should be on average $b \sim-3^{\circ}$. However, the presence of a plane of symmetry at $b \sim-3^{\circ}$ is not what M06 meant. Moreover, the well-known agemetallicity-distance degeneracy can also introduce some minor quantitative error in any interpretation of CMa CMDs. In the 


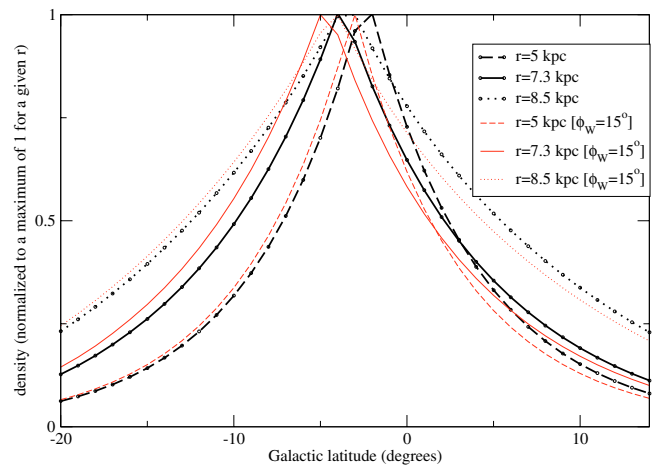

Fig. 1. Stellar density (normalized to a maximum of unity) as a function of galactic latitude for $l=240^{\circ}$ and source distance at 5.0, 7.3 and $8.5 \mathrm{kpc}$ respectively, according to the L06 Galactic model (with $\phi_{W}=+5^{\circ}$ ), or the L06 model with $\phi_{W}=+15^{\circ}$ (as derived by M06). This figure shows that intercepting the disc at symmetric latitudes with respect to the local mid-warp, one still obtains stellar densities with different profiles.

following paragraphs we show why a CMD comparison at two given latitudes is not straightforward.

It is crucial to understand that the asymmetries introduced by the warp change for different values of distance from the Sun $(r)$, and that this implies different densities as a function of the absolute magnitudes of the main sequence stars. To quantify this effect we make use of the L06 warp model $^{1}$ and calculate in Fig. 1 the stellar density (normalized to unity in each case) as a function of Galactic latitude for different distances. If we use a line of nodes angle of $\phi_{W}=15^{\circ}$ (M06) instead of $\phi_{W}=5^{\circ}$ (L06) the results remain similar except for a slight shift of 1-2 degrees in the latitude of maximum density. Thus, Fig. 1 shows that the warp asymmetries are a function of the heliocentric distance and thereby neither the overall star counts nor the general features of the CMD are expected to be similar in the $b \approx+8^{\circ}$ and $b \approx-15^{\circ}$ or $b \approx+4^{\circ}$ and $b \approx-9^{\circ}$ diagrams.

Indeed, one should keep in mind that any point (colourmagnitude pair) of a CMD displays an integration of all detected stellar populations along the line of sight (i.e. at all distances) for each CMDs pair. One cannot isolate (via visual inspection or isochrone superposition) the $\sim 7.2 \mathrm{kpc}$ stellar populations in these diagrams. The isochrone plotting in Fig. 28 of C07 may show a higher stellar density at distances of $\sim 7.2 \mathrm{kpc}$. Nevertheless, presumed CMa sequences may include stars at nearer distances and one cannot isolate the $\mathrm{CMa}$ populations from young, faint and nearby main-sequence stars. This precludes any quantitative conclusion on the non-similarity of CMDs pairs at CMa distances. Integrating along the $b \approx$ $-15^{\circ}$ line of sight one clearly "travels" below the stellar disc. However, assuming that the disc is warped downwards in these directions, one will sample more disc stars and might even intercept the warping disc at a certain scale height. This is the opposite for the $b \approx+8^{\circ}$ field where, integrating along this line of sight, one will sample less and less of the warped disc and, most importantly, will not intercept a significant portion of its scale height in the III quadrant. The sudden appearance of a seemingly separated main sequence in the $b \approx-15^{\circ}$ field is possibly the signature of the warp.

One should also keep in mind the unknown but important uncertainties due to possible variations of the reddening law

1 The L06 warp model is the same as the López-Corredoira et al. (2002) model, except for a $\phi_{W}$ of $+5^{\circ}$ and an extrapolated $z_{W}(13 \mathrm{kpc}<$ $R<16 \mathrm{kpc})=z_{W}(13 \mathrm{kpc})$ for the southern warp. while crossing the warped mid-plane at large distances. The left panel of Fig. 9 in B07 shows two vertical lines that delimit the "high reddening region": $E_{(B-V)} \geq 0.30$. This sketches the strong downward disc warping as traced by the interstellar dust. This dust asymmetry of $\sim 2-3^{\circ}$ is consistent with that found for the gas and stars (M06). Interestingly, a hint that the stellar disc shows a $\sim 2-3^{\circ}$ downward bending in this line of sight is found by B07. The total extinction for $b \approx+8^{\circ}$ and $b \approx-15^{\circ}$ or $b \approx+4^{\circ}$ and $b \approx-9^{\circ}$ is similar, however the differential reddening is expected to be quite different (again, because the lines of sight follow very different paths): there is a different reddening as a function of the magnitude that therefore produces different apparent shapes of the main sequence.

Thus, there is nothing anomalous in CMDs being equally displaced in latitude from the $b \approx-3^{\circ}$ warped mid-plane being different: integration along the line of sight is not symmetric, and the warp is not asymmetric for all heliocentric distances, and neither is extinction.

\subsection{Distance of the maximum overdensity produced by the warp}

B07 make use of a modified L06 warp model ${ }^{2}$ and infer that the peak of the star counts, for a given population of stars along the CMa line of sight, is at $(m-M) \approx 10.5$ (i.e. a distance $d=$ $1.3 \mathrm{kpc}$ ). This value is at odds with a correct application of L06, and implies a serious analysis error by B07.

Leaving aside the extinction, the star counts for a stellar population with magnitude $M$ up to $m$, having a density distribution of $\rho(\boldsymbol{r})$ and certain $(l, b)$ Galactic coordinates within an area of $\omega$ radians can be expressed as follows:

$$
\begin{aligned}
& N(m)=\omega \int_{0}^{r(m)} \rho[x, l, b] x^{2} \mathrm{~d} x, \\
& r(m)=10^{[m-M+5] / 5} \text { (distance), }
\end{aligned}
$$

rather than $N(m)=\omega \int_{0}^{r} \rho x \mathrm{~d} x$, as used by B07 (Peñarrubia, priv. comm., Oct.-2006, who also performed the B07 calculations), based on Eqs. (7) and (8) of Peñarrubia et al. (2005). We believe that the Peñarrubia formulation is incorrect because the counts per magnitude interval are not equal to the counts per unit distance (it is not correct to use $N(m)=N(r)$ in Eq. (7) of Peñarrubia et al. (2005)). Thus, it is straightforward to obtain:

$\frac{\mathrm{d} r(m)}{\mathrm{d} m}=\frac{\ln 10}{5} r(m)$

and consequently the counts at magnitude $m$ per unit magnitude are

$A(m) \equiv \frac{\mathrm{d} N(m)}{\mathrm{d} m}=\frac{\ln 10}{5} \omega \rho[r(m), l, b] r(m)^{3}$,

rather than $\frac{\mathrm{d} N}{\mathrm{~d} m} \propto \rho r$ as used in B07 (Peñarrubia, priv. comm. Oct.-2006). Should one apply this last (incorrect) formula, one would derive a distance of the maximum warp overdensity at $(m-M) \approx 11.1$. There is an $(m-M) \approx 0.6$ difference between our reconstruction of the erroneous calculations of B07 and their reported value, and this is probably due to the inclusion of extinction in the B07 calculations. B07's erroneous calculation most probably stems from this wrong application of the stellar statistics equation (see also errors listed in L06 (Sect. 2.3)).

2 They take constant height at $R>14 \mathrm{kpc}$ instead of $R>13 \mathrm{kpc}$, and $\phi_{W}=-5^{\circ}$ instead of $\phi_{W}=+5^{\circ}$. These parameters, however, do not affect the present comments. 


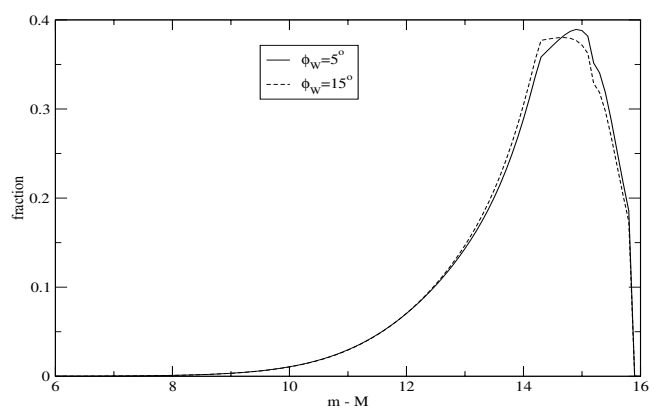

Fig. 2. The fraction of stars (normalized to give an area of unity) as a function of $(m-M)$ for $(l, b)=\left(241.5^{\circ},-7.5^{\circ}\right)$ predicted by the L06 warp-model (assuming zero extinction along the line of sight). The short-dashed line is obtained with a modified L06 model with $\phi_{W}=15^{\circ}$.

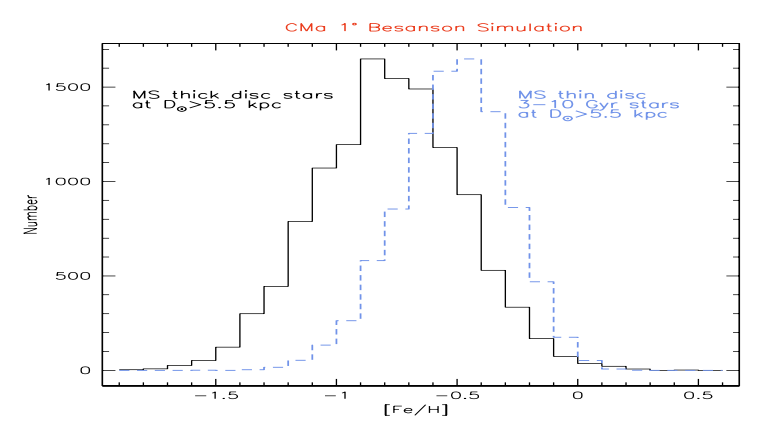

Fig. 3. A $1^{\circ}$ Besançon simulation along the CMa centre line of sight showing the expected thin and thick Galactic disc metallicities. The dashed histogram (main sequence thin disc stars) has been normalized to that of the thick disc.

Not surprisingly, several authors (Bellazzini et al. 2006b; M06; L06) have already demonstrated that the correct distance is in the range 5-10 kpc. Figure 2 shows our application of the L06 warp model and shows a clear disagreement with Fig. 14 of B07. In our case, and assuming zero extinction, the maximum of the star counts (proportional to $\left.\rho(r) r^{3}\right)$ is found at $(m-M) 。 14.9$, a distance of $\sim 10 \mathrm{kpc}$. Indeed, this result is implicit in L06 (Fig. 2): since the peak of the red clump stars was found around $m_{K}=13.3$, this corresponds to $(m-M) \approx 15$ (considering that $\left.M_{K \text {,red clump }} \approx-1.65\right)$. On the other hand, the total extinction for the CMa centre is around $A_{B}=0.99$ (Schlegel et al. 1998). When this is taken into account, the resulting maximum should be at $\left(m_{B}-M_{B}\right)>13.9$, depending slightly on the absolute magnitude $M_{B}$ of the adopted population.

Our Fig. 2 shows that the B07 star-count maximum (their Fig. 14) is in agreement with the L06 warp model predictions. There is a small difference concerning the depth of the main sequence star-count maximum $(F W H M)$. This is predicted around 2.2 mag (see also L06 (Sect. 2.3; Fig. 3)) whereas the B07 observed depth is $30 \%$ lower (L06, Sect. 2.3). This is a natural effect of inaccuracies in the warp+flare modelling. One should, however, keep in mind that extinction would contribute towards narrowing the $F W H M$, thus reducing the disagreement between the warp model expectations and observations. This is particularly true if an important fraction of the total extinction, along the line of sight, is associated with distances near the CMa overdensity. Either way, the warp model-observation differences are too small to justify the exclusion of the warp hypothesis and the need for an extra (accreted) population.

One might wonder why we argue that our first-order Galactic model solves for the distribution of the observed overdensity while we argue that other models like Besançon
(Robin et al. 2003, extensively used by C07 and B07) do not. The Besançon model has been tested (C07 and $\mathrm{B} 07)$ and provides an excellent description of the Galaxy in many lines of sight. But the Besançon model does not include a lower amplitude of the southern stellar warp with respect to the northern one (L06). Moreover, the model does not include the CMa young population of stars associated with the Norma-Cygnus spiral arms (see next subsection). Therefore, distentangling different stellar sequences in CMa CMDs (via galactic model comparisons) is intrinsically difficult, even before investigating the presence of a dwarf galaxy.

\subsection{CMa blue plume population}

Colour-magnitude diagrams of the centre of CMa show the conspicuous presence of stars brighter than the old MS turnoff. Bellazzini et al. (2004) were the first to interpret this population as 1-2 Gyr CMa stars, arguing that the CMa stellar overdensity (originally identified by older red clump stars) also shows an overdensity of young stars. However, the same blue plume population was explained (Carraro et al. 2005; Moitinho et al. 2006) in terms of a much younger $(\sim 100 \mathrm{Myr})$ thin disc spiral arm and inter-spiral population. The lack of a blue plume population was noted in all the B07 fields above the mid-plane, and in fields between $193^{\circ} \leq l \leq 220^{\circ}$ and below the mid-plane. This leaves little space for a unique $\mathrm{CMa}$ blue plume population connection and, admittedly, C07 favour a Galactic origin of the blue plume population.

An erroneous approach of B07 or d07 is to make a distinction between the warp+flare (M06, L06) and the spiral arm (Carraro et al. 2005 and Moitinho et al. 2006) scenarios. The warp+flare and spiral arm interpretations are complementary, as they refer to (young and old) stellar populations having the same Galactic origin. The Milky Way disc populations, regardless of their age, do warp. Spiral arms warp downwards in the 3rd quadrant of the external disc. Consequently, an excess of blue plume stars appears when one observes along southern Galactic latitude lines of sight. Indeed, the blue plume population survey by Moitinho et al. (2006, the lower panel of their Fig. 2) shows a clear sign of warping for the young stellar populations in relation to the CMa distance. A successive wide-field III quadrant survey by Carraro et al. (2007) confirms the presence of a warped young stellar populations at about $\sim 9 \mathrm{kpc}$, associated with the Norma-Cygnus spiral arm. Even more interestingly, Carraro et al. (2007) trace the presence of an older ( $\sim 7 \mathrm{Gyr})$ population and identify its MS and red giant stars. They conclude that this $\sim 7 \mathrm{Gyr}$ population is associated with the old and warped thin/thick disc component.

Thus, the "CMa blue plume population" is most likely to reflect a warped Milky Way thin disc population, as those with older ages; no conspiracy of two different effects as said by B07 or $\mathrm{d} 07$, just warping disc populations.

\subsection{CMa extension, disruption and tidal effects}

In their Fig 9, B07 shows that there is little surface density gradient in longitude across their CMa survey area. In particular, there is a clear density profile compatibility between the red clump (based on 2MASS star counts by Bellazzini et al. 2006b) and the old main sequence (based on the B07 optical survey) studies. A near-flat profile over $\sim 30^{\circ}$ implies a higher elongation and weakens the definition of an "overdensity". This is not a minor detail in the CMa debate because the CMa stellar populations (thought of as an extra population) would actually 
connect up with the Argo overdensity (Rocha-Pinto et al. 2006: $l \sim 300^{\circ}$ ). If one accepts an extra-Galactic origin for such a huge and elongated overdensity (covering an extension larger than the entire III quadrant) it is legitimate to ask: where is the "Galactic" stellar disc?

For the old CMa main sequence population, B07 estimate a $F W H M_{(l, b)}=\left(>27^{\circ}, \sim 6^{\circ}\right)$ and a ratio of $>5: 1$. On the other hand, for the young CMa main sequence population they derive $F W H M_{(l, b)}=\left(>20^{\circ}, \sim 2^{\circ}\right)$ and a ratio of $>10: 1$, i.e. the most recent stars formed at the centre would have been the last to be stretched, which is against what is expected in a dwarf galaxy. Interestingly, the young main sequence density profile (B07, Fig. 9, right panel) shows a peak in their longitude coverage. This can easily fit the scenario in which the Galactic stellar disc (in the CMa line of sight) follows the trend of the warped gas: starts warping downwards, reaches a maximum at Galactocentric distances of $\sim 13 \mathrm{kpc}$ ( $\sim$ the CMa distance), and then re-approaches the nominal Galactic mid-plane. Should this be the case, then the peak in the young main sequence density profile at $240^{\circ}$ would correspond to the "interception" of the thin disc warping downwards.

The effect of a dwarf galaxy or massive substructure with an extension of several kpc embedded in the Milky Way at only $13 \mathrm{kpc}$ of the Galactic centre should present distortions of the Galactic disc and spiral arms, which are not observed. Moreover, and as discussed in B07, while orbiting the Galactic centre, a disrupting coplanar satellite would be subject to continuous tidal forces that give rise to tidal tails. Any bound portion should not, however, show a flat density distribution, as found for CMa.

\subsection{The CMa metallicity}

Recently, D07 presented a quantitative analysis of wide-field CMDs in and around the CMa centre. Their isochrone fitting technique suggests a metallicity, $[\mathrm{Fe} / \mathrm{H}]$, of between $-0.6 \pm 0.3$ for the fields that are further away from the plane and $-1.0 \pm 0.2$ (D07; Table 2) for the fields that are closer to the plane (and consequently with larger problems with extinction). Bellazzini et al. (2006a) with a similar method had reported $-0.7<[M / H]<$ -0.4 near the centre of the Canis Major overdensity. D07 also anticipate FLAMES and AAOMEGA spectroscopic metallicity results (by Martin et al., in prep.) of kinematically selected members showing $[\mathrm{Fe} / \mathrm{H}] \approx-0.9$. This leads D07 to argue against the warp/flare hypothesis, with which we do not agree.

Leaving aside the uncertainties (rough extinction assumptions, foreground/background contamination and distancemetallicity degeneracy) that affect the D07 analysis, it is interesting to note, again, that there is nothing strange in this "low" metallicity within our Galaxy. This is shown in the bimodal distribution of Fig. 3, the expected Milky Way thin and thick disc metallicities (from a Besançon simulation for old main sequence stars; Robin et al. 2003). From a more general point of view, Hammer et al. (2007) observe that the chemical abundance of the Milky Way outskirts is three times lower than those of most spiral galaxies within a similar mass range. Added to a smaller stellar mass and angular momentum, Hammer et al. infer an exceptionally quiet formation history for the Milky Way, apparently escaping any significant merger over the past $\sim 10$ Gyr. Chemical abundances (Sbordone et al. 2005) suggest that some CMa regions might have experienced some unusual star formation, but it is at present inconclusive about CMa origin.

\section{Discussion and conclusions}

We re-examined the most recent claims $(\mathrm{C} 07, \mathrm{~B} 07$ and d07) in favor of an accreted dwarf galaxy in Canis Major. In particular, we unambiguously show (Fig. 2) that the Galactic warp produces an overdensity whose maximum coincides in distance with that reported for CMa. We show that contrary claims were based on erroneous calculations. We also argue that the warp and the spiral arms/segments scenarios should not be put forward as two different explanations. Young and old Galactic components, equally, warp. Lastly, we argue that a low metallicity for $\mathrm{CMa}$ populations is not at odds with typical thick disc populations.

As it stands, we cannot see any property of the CMa stellar overdensity that cannot be accounted for in terms of a "smoothly" warped and flared Galactic stellar disc. On the contrary, the lack of ancient stellar populations ${ }^{3}$, a compatibility of CMa radial velocities, proper motion, and chemical composition with typical disc values indicate the Galactic origin of the overdensity.

Acknowledgements. Thanks are given to the referee Blair Conn (ESO) for very helpful comments. M. López-Corredoira was supported by the Ramón y Cajal Programme of the Spanish Science Ministry. This research has partially been supported by the Italian INAF PRIN grant CRA 1.06.08.02.

\section{References}

Bellazzini, M., Ibata, R., Monaco, L., et al. 2004, MNRAS, 354, 1263 Bellazzini, M., Correnti, M., Ferraro, F. R., Monaco, L., \& Montegriffo, P. 2006a, A\&A, 446, L1

Bellazzini, M., Ibata, R., Martin, N., et al. 2006b, MNRAS, 366, 865 Butler, D. J., Martínez-Delgado, D., Rix, H.-W., Peñarrubia, J., \& de Jong, J. T. A. 2007, AJ, 133, 2274 (B07)

Carraro, G., Vázquez, R., Moitinho, A., \& Baume, G. 2005, ApJ, 630, L153

Carraro, G., Moitinho, A., Zoccali, M., Vázquez, R., \& Baume, G. 2007, AJ 133, 1058

Conn, B. C., Lane, R. R., Lewis, G. F., et al. 2007, MNRAS, 376, 939 (C07) de Jong, J. T. A., Butler, D. J., Rix, H.-W., Dolphin, A. E., \& Martínez-Delgado, D. 2007, ApJ, 662, 259 (d07)

Hammer, F., Puech, M., Chemin, L., Flores, H., \& Lehnert, M. 2007, ApJ, 662, 322

López-Corredoira, M. 2006, MNRAS, 369, 1911 (L06)

López-Corredoira, M., Cabrera-Lavers, A., Garzón, F., \& Hammersley, P. L. 2002, A\&A, 394, 883

Martin, N. F., Ibata, R. A., Bellazzini, M., et al. 2004, MNRAS, 348, 12

Mateu, C., Vivas, A. K., Zinn, R., \& Miller, L. 2007, [arXiv:astro-ph/0702115]

Moitinho, A., Vázquez, R. A., Carraro, G., et al. 2006, MNRAS, 368, 77 Momany, Y., Zaggia, S. R., Bonifacio, P., et al. 2004, A\&A, 421, L29

Momany, Y., Zaggia, S. R., Gilmore, G., et al. 2006, A\&A, 451, 515 (M06) Peñarrubia, J., Martínez-Delgado, D., Rix, H. W., et al. 2005, ApJ, 626, 128 Robin, A., Reylé, C., Derrire, S., \& Picaud, S. 2003, A\&A, 509, 523

Rocha-Pinto, H. J., Majewski, S. R., Skrutskie, M. F., et al. 2006, ApJ, 640, L147 Sbordone, L., Bonifacio, P., Marconi, G., Zaggia, S., \& Buonanno, R. 2005, A\&A, 430, L13

Schlegel, D. J., Finkbeiner, D. P., \& Davis, M. 1998, ApJ, 500, 525

\footnotetext{
3 The small number of detected RR Lyrae stars in the CMa overdensity can be accounted for by the halo and thick disc RR Lyrae population (Mateu et al. 2007).
} 